\title{
Study of somesthesis according to change in pulse diode laser parameters
}

\author{
Ji-Sun Kim, Han-Byeol Oh, A-Hee Kim, Jun-Sik Kim, Eun-Suk Lee, Bong-Jun Goh, Tae-Hee \\ Lee, Soon-Cheol Chung and Jae-Hoon Jun* \\ Department of Biomedical Engineering, BK21 Plus Research Institute of Biomedical Engineering, \\ College of Biomedical and Health Science, Konkuk University, Chungju, Republic of Korea
}

\begin{abstract}
Laser can precisely deliver quantitative energy to a desired region in a non-contact way. Since it can stimulate regions and minutely control parameters such as the intensity, duration and frequency of stimulus, laser is often used for the areas such as low power laser treatment and clinical physiology. This study proposes simulation using pulse diode laser with reliable output and identifies laser parameters that can present a variety of somesthesis. It is found that typically, as frequency and energy increase, the ratio of feeling senses increases, and dominant sense moves from the sense of heat through tactile sense to pain. This study will be baseline data for studies of the sense of heat, tactile sense and pain, contribute to studying neurophysiology sector and be applied to basic clinical research.
\end{abstract}

Keywords: Biostimulation, heat and pain sense, laser beam physical parameters, laser-tissue interaction, tactile

\section{Introduction}

For many decades, laser technology has developed in many fields such as industry, biology, and medicine because of the unique characteristic of the laser [1,2]. As an acronym of Light Amplification by Stimulated Emission of Radiation, laser is a very special light source. Laser can emit 'artificial light with even direction, phase and wavelength' differentiated from natural light, and based on this characteristic, it is used in many applications. Specifically, it is used in a wide range of industries covering: 1) Optical communications area using optical characteristics; 2) Medical areas such as disease monitoring, low level laser therapy and Photo Dynamic Therapy (PDT) [3]; 3) Nanotechnology area separating chemical bonding; and 4) Precision machine tools like diamond processing etc [4].

Laser has a merit that it does not have any direct contact with the skin when it presents a stimulus. Thus, laser is often used in a study of nociceptor and has a non-contact characteristic, and it can present somesthesis in a safe state while maintaining its own characteristic [5]. Especially, the method using laser can present a certain stimulus in the right place repeatedly and has a very high stimulus resolution $[6,7]$. Thus, it can be used in the areas of the senses of heat or pain, or cell stimulus

\footnotetext{
* Address for correspondence: Jae-Hoon Jun, Department of Biomedical Engineering, College of Biomedical and Health Science, Konkuk University, 268 Chungwondaero, Chungju, Chungbuk, 380-701, Republic of Korea, Tel.: +82 438403799 ; Fax: +82 43 8454814; E-mail: jjun81@kku.ac.kr.
} 
experiment [8-12]. After the incidence of laser beam into the skin, the optical energy distribution in the skin changes by optical coefficients such as absorption coefficient, scattering coefficient, refractive index, and anisotropy factor [13]. Laser acts as an energy source, and the energy that has come into the skin tissue is converted to thermal energy by thermal transfer coefficients (conductivity, heat capacity, convective coefficients, and emissivity of the tissue), the temperature increases in the living body, and the heat is transferred to the surrounding tissues. At this time, thermos receptive detects thermal sensation, the repetition rate of action potential begins to increase at about $30^{\circ} \mathrm{C}$ and keeps increasing up to about $45^{\circ} \mathrm{C}$. At over $45^{\circ} \mathrm{C}$, the repetition rate decreases rapidly, and from this time, action potential of temperature-related thermal nociceptor begins to be generated, and it is perceived from warm and hot to scalding, which is classified into the area of pain $[14,15]$. Previously, there are methods of presenting the sense of heat, such as one using peltier module; one using high frequency stimulator; and one using heat stimulator. To look into each, peltier module produces the sense of heat stimulus according to the magnitude of the voltage, but the temperature should be compensated with an analog temperature sensor, and there is a limit in producing thermal sensation presented modules in a free form. A high frequency stimulator generates heat as the molecules vibrate with the addition of electric energy by abrasion, but sparks may occur and the method of use is complicated. In addition, heat stimulation consists of a thermal stimulator at a constant temperature, but the size of stimulus is not controlled, so it has a demerit that it is difficult to deliver thermal stimulus to regions. Current technology for tactile stimulus presentation uses mechanical, electrical or thermal tactile stimulator, so it has a limit in quantitatively controlling parameters such as the intensity, presenting area and duration of stimulus precisely.

Thus, this study conducts the experiment using diode laser with stable output, and of which the laser parameters can be changed precisely. This study aims to identify tactile sense using in a non-contact method. Based on this, the laser energy by which the senses of heat and pain feel is presented, and the tendency is observed. This study identifies the range causing tactile sense by changing various laser parameters such as energy, frequency and beam diameter. This study has a merit that it is noncontacting, and it can quantitatively control stimulus by controlling energy per unit pulse. It is judged that this study will contribute to studying the senses of heat and pain in clinical area or neurophysiology sector.

\section{Material and methods}

\subsection{Laser system}

Figure 1 shows the laser used in the experiment, a fiber coupled diode laser system (PearlTMTKS-B, nLight Photonics Corp., Santa Clara, CA), with a merit that it is easy to control various laser parameters. Fiber core diameter is $400 \mu \mathrm{m}$, and Numerical Aperture (NA) is 0.22 . Wavelength is 809 $\mathrm{nm}$; pulse width (Full-Width at Half-Maximum: FWHM) is varying from $10 \mu \mathrm{s}$ to $\mathrm{CW}$; repetition rate is $<12.5 \mathrm{kHz}$; and maximum output peak power is $35 \mathrm{~W}$. The laser beam delivered to optical fiber is diverged according to the core diameter and NA of the optical fiber, so it is constructed such that the beam can be easily operated using a collimating lens. Beam diameter is precisely controlled so that it is focused on the finger by using a convex lens $(\mathrm{f}=38.1 \mathrm{~mm})$ and a 3-axis translation stage. Laser pulse energy and pulse width can be measured with a pyroelectric energy detector (818E-05-12-L, Newport, Irvine, CA) and an energy meter (1918-R, Newport, Irvine, CA), respectively, and the beam diameter is measured using a beam profiler (SP620U, Ophir Optronics Inc., Danvers, MA) at the 


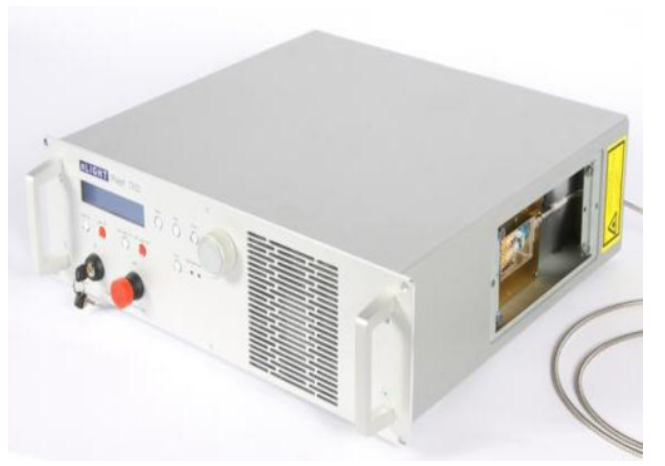

Fig. 1. Diode laser used in the experiment.

Table 1

Laser parameters used in the experiment

\begin{tabular}{ll}
\hline Experiment variable & Experiment range \\
\hline Energy & $150 \sim 307 \mu \mathrm{J}(8 \mathrm{steps})$ \\
\hline Pulse width & $10 \mu \mathrm{s}$ \\
\hline Frequency & $1,2,3,3.5,4,4.5,5,5.5[\mathrm{kHz}]$ \\
\hline Episode time & $5 \mathrm{~s}(\mathrm{Stop}$ experimentation if a pain occurs $)$ \\
\hline Beam diameter & $0.46,1.86,6.7 \mathrm{~mm}(1 / \mathrm{e})$ \\
\hline Subjects & A total 20 persons $(14$ males +6 females $)$ \\
\hline
\end{tabular}

position of the finger to which the laser is irradiated.

\subsection{Stimulus characterization}

Table 1 is the one that summarizes the experiment variables. Subjects consist of 20 persons, 14 males and 6 females, who are all right-handed. The subjects cannot perceive that the stimulating device is laser, and the distribution of their age ranges between 19 and 25. Laser energy $(150 \mu \mathrm{J} \sim 307$ $\mu \mathrm{J})$ was irradiated, and pulse width was fixed at $10 \mu \mathrm{s}$. The episode time of laser irradiation was $5 \mathrm{sec}$ at a frequency of $1 \mathrm{kHz} \sim 5.5 \mathrm{kHz}$ and the subjects' tactile responses were recorded. This experiment was approved by the Institutional Review Board at Konkuk University (7001355-201408-HR-032).

Figure 2 shows the experimental setup for laser tactile stimulation. In checking the tactile responses, the subjects were asked a question regarding what it felt and asked to deliver their feelings freely. The

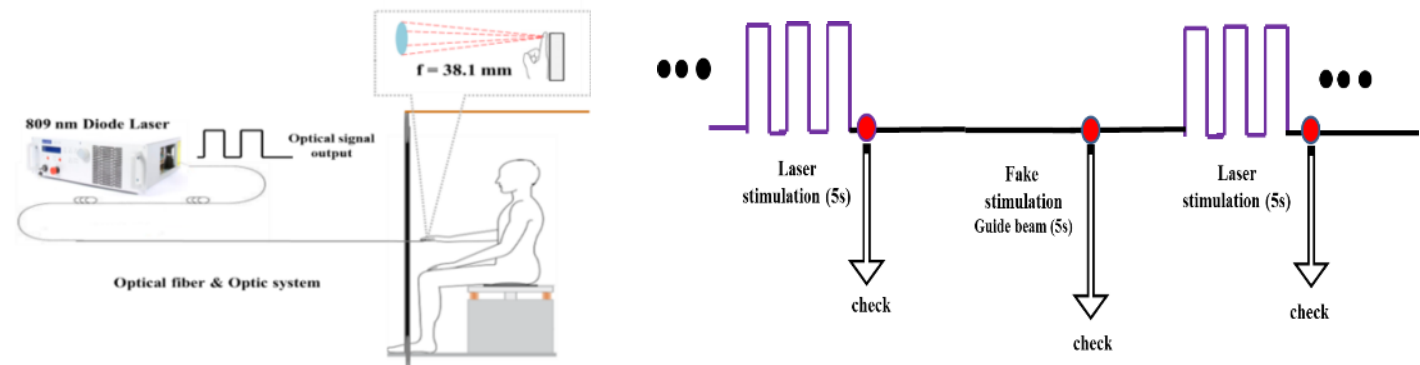

Fig. 2. Method of laser stimulation. 
Table 2

Results of behavioral responses by the laser energy and frequency change

\begin{tabular}{lllllll}
\hline \multicolumn{2}{c}{ Frequency } \\
Energy & $1 \mathrm{kHz}$ & $2 \mathrm{kHz}$ & $3 \mathrm{kHz}$ & $3.5 \mathrm{kHz}$ & $4 \mathrm{kHz}$ & $4.5 \mathrm{kHz}$ \\
\hline $150 \mu \mathrm{J}$ & $0 \%$ & $15 \%$ & $20 \%$ & $30 \%$ & $45 \%$ & $40 \%$ \\
\hline $160 \mu \mathrm{J}$ & $0 \%$ & $20 \%$ & $25 \%$ & $30 \%$ & $35 \%$ & $55 \%$ \\
\hline $200 \mu \mathrm{J}$ & $0 \%$ & $15 \%$ & $35 \%$ & $45 \%$ & $55 \%$ & $65 \%$ \\
\hline $226 \mu \mathrm{J}$ & $0 \%$ & $20 \%$ & $40 \%$ & $55 \%$ & $60 \%$ & $85 \%$ \\
\hline $245 \mu \mathrm{J}$ & $10 \%$ & $20 \%$ & $50 \%$ & $55 \%$ & $65 \%$ & $100 \%$ \\
\hline $258 \mu \mathrm{J}$ & $10 \%$ & $30 \%$ & $40 \%$ & $60 \%$ & $75 \%$ & $100 \%$ \\
\hline $278 \mu \mathrm{J}$ & $5 \%$ & $30 \%$ & $65 \%$ & $100 \%$ & $95 \%$ & $100 \%$ \\
\hline $307 \mu \mathrm{J}$ & $20 \%$ & $35 \%$ & $90 \%$ & $100 \%$ & $100 \%$ & $100 \%$ \\
\hline
\end{tabular}

\begin{tabular}{ll}
\hline No sense of more than $50 \%$ \\
\hline Sense of more than & Heat \\
\cline { 2 - 2 } $50 \%$ & Tactile \\
\cline { 2 - 2 } & Pain \\
\hline
\end{tabular}

experiment used black tents at four corners, and used white noise to cut off external senses such as vision and auditory sense, so that they could focus on their tactile sense only. Also, the subjects were not allowed to perceive the equipment that would provide stimulus was a laser in advance, and a fake stimulus was inserted randomly between the laser stimuli, and by checking if the subjects could perceive that when no actual stimulus was given, the experiment was conducted only when they could differentiate the fake stimulus accurately. In addition, the beam diameter was changed from $0.46 \mathrm{~mm}$ to $6.7 \mathrm{~mm}$, so that the responses with the beam diameter could be checked.

\section{Results and discussion}

Table 2 shows the behavioral responses by the energy and frequency change when the beam diameter is $0.46 \mathrm{~mm}$. The result of experiment showed the section that more than $50 \%$ of subjects felt sense by changing the frequency and energy. The subjects did not feel any sense when the frequency was the period of less than $1 \mathrm{kHz}$. The inner numerical value of Table 2 shows the ratio (Ratio of adding all sense of heat, tactile, pain) that the subjects felt the senses, and in the range that more than $50 \%$ of subjects felt sense, the most dominant sense was marked by color. The tactile means the mechanical feeling but the sense of heat nor pain, and the subjects expressed it as pricking, stinging, pressing etc.

The part filled with yellow is the area of the dominant sense of heat, and the part filled with green color is the area of the dominant sense of tactile, and the part filled with red means the area of dominant pain. In the same energy, the higher frequency shows the more rate of sense increased, and the most of sense was shown in the order of heat, tactile, and pain. Most of the subjects who answered tactile in the result of experiment expressed pricking, and there were stinging, pressing etc. as the other tactile response. In most of the cases, when the energy increase in the same frequency condition, the ratio of sense increased, and equally, when the frequency increased in the same energy condition, the ratio of sense increased as well. Particularly, most of subjects answered the pain when the energy was more than $258 \mu \mathrm{J}$, and the frequency was more than $4 \mathrm{kHz}$.

Table 3 shows the result of experiment about the sense of heat, tactile, and pain in the condition with different beam diameter. Table 3(a) shows the experiment result when the beam diameter was $0.46 \mathrm{~mm}$, Table $3(\mathrm{~b})$ shows the experiment result when the beam diameter was $1.86 \mathrm{~mm}$, and Table 3(c) shows the experiment result when the beam diameter was $6.7 \mathrm{~mm}$. The purpose of the experiment by increasing beam diameter is to examine the effect of energy density change. When the beam diameter is $0.46 \mathrm{~mm}$ as the experiment condition, there was no dominant sense area under the 
Table 3

Laser parameter condition when the sense of heat, tactile, pain appear

(a) Beam diameter $(0.46 \mathrm{~mm})$

\begin{tabular}{lllll}
\hline \multirow{2}{*}{$\begin{array}{l}\text { Energy } \\
150 \mu \mathrm{J}\end{array}$} & $3 \mathrm{kHz}$ & $3.5 \mathrm{kHz}$ & $4 \mathrm{kHz}$ & $4.5 \mathrm{kHz}$ \\
\hline $160 \mu \mathrm{J}$ & $20 \%$ & $30 \%$ & $45 \%$ & $40 \%$ \\
\hline $200 \mu \mathrm{J}$ & $25 \%$ & $30 \%$ & $35 \%$ & $55 \%$ \\
\hline $226 \mu \mathrm{J}$ & $35 \%$ & $45 \%$ & $55 \%$ & $65 \%$ \\
\hline $245 \mu \mathrm{J}$ & $40 \%$ & $55 \%$ & $60 \%$ & $85 \%$ \\
\hline $258 \mu \mathrm{J}$ & $50 \%$ & $55 \%$ & $65 \%$ & $100 \%$ \\
\hline $278 \mu \mathrm{J}$ & $40 \%$ & $60 \%$ & $75 \%$ & $100 \%$ \\
\hline $307 \mu \mathrm{J}$ & $65 \%$ & $100 \%$ & $95 \%$ & $100 \%$ \\
\hline
\end{tabular}

\begin{tabular}{ll}
\hline No sense of more than $50 \%$ \\
\hline Sense of more than & Heat \\
\cline { 2 - 2 } $50 \%$ & Tactile \\
\cline { 2 - 2 } & Pain \\
\hline
\end{tabular}

(b) Beam diameter $(1.86 \mathrm{~mm})$

\begin{tabular}{lllllll}
\hline $\begin{array}{l}\text { Erequency } \\
\text { Energy }\end{array}$ & $3 \mathrm{kHz}$ & $3.5 \mathrm{kHz}$ & $4 \mathrm{kHz}$ & $4.5 \mathrm{kHz}$ & $5 \mathrm{kHz}$ & $5.5 \mathrm{kHz}$ \\
\hline $150 \mu \mathrm{J}$ & $5 \%$ & $5 \%$ & $25 \%$ & $30 \%$ & $25 \%$ & $30 \%$ \\
\hline $160 \mu \mathrm{J}$ & $10 \%$ & $30 \%$ & $40 \%$ & $55 \%$ & $40 \%$ & $65 \%$ \\
\hline $200 \mu \mathrm{J}$ & $20 \%$ & $40 \%$ & $40 \%$ & $55 \%$ & $70 \%$ & $65 \%$ \\
\hline $226 \mu \mathrm{J}$ & $25 \%$ & $35 \%$ & $55 \%$ & $55 \%$ & $65 \%$ & $80 \%$ \\
\hline $245 \mu \mathrm{J}$ & $25 \%$ & $35 \%$ & $70 \%$ & $80 \%$ & $80 \%$ & $95 \%$ \\
\hline $258 \mu \mathrm{J}$ & $25 \%$ & $50 \%$ & $65 \%$ & $75 \%$ & $80 \%$ & $95 \%$ \\
\hline $278 \mu \mathrm{J}$ & $30 \%$ & $60 \%$ & $75 \%$ & $85 \%$ & $95 \%$ & $95 \%$ \\
\hline $307 \mu \mathrm{J}$ & $55 \%$ & $75 \%$ & $80 \%$ & $90 \%$ & $95 \%$ & $95 \%$ \\
\hline
\end{tabular}

(c) Beam diameter $(6.7 \mathrm{~mm})$

\begin{tabular}{lllllll}
\hline $\begin{array}{r}\text { Erequency } \\
\text { Energy }\end{array}$ & $3 \mathrm{kHz}$ & $3.5 \mathrm{kHz}$ & $4 \mathrm{kHz}$ & $4.5 \mathrm{kHz}$ & $5 \mathrm{kHz}$ & $5.5 \mathrm{kHz}$ \\
\hline $150 \mu \mathrm{J}$ & $0 \%$ & $0 \%$ & $0 \%$ & $0 \%$ & $5 \%$ & $10 \%$ \\
\hline $160 \mu \mathrm{J}$ & $0 \%$ & $0 \%$ & $0 \%$ & $5 \%$ & $15 \%$ & $20 \%$ \\
\hline $200 \mu \mathrm{J}$ & $0 \%$ & $0 \%$ & $0 \%$ & $5 \%$ & $15 \%$ & $25 \%$ \\
\hline $226 \mu \mathrm{J}$ & $5 \%$ & $5 \%$ & $5 \%$ & $10 \%$ & $20 \%$ & $35 \%$ \\
\hline $245 \mu \mathrm{J}$ & $5 \%$ & $5 \%$ & $5 \%$ & $20 \%$ & $35 \%$ & $40 \%$ \\
\hline $258 \mu \mathrm{J}$ & $5 \%$ & $5 \%$ & $15 \%$ & $30 \%$ & $30 \%$ & $35 \%$ \\
\hline $278 \mu \mathrm{J}$ & $10 \%$ & $15 \%$ & $15 \%$ & $30 \%$ & $35 \%$ & $35 \%$ \\
\hline $307 \mu \mathrm{J}$ & $15 \%$ & $30 \%$ & $35 \%$ & $40 \%$ & $40 \%$ & $45 \%$ \\
\hline
\end{tabular}

frequency $3 \mathrm{kHz}$ (see Table 2). Therefore, the additional experiment was conducted in the frequency range of more than $3 \mathrm{kHz}$, and more experiments were conducted at $5 \mathrm{kHz}$ and $5.5 \mathrm{kHz}$.

By comparing Tables 3(a) and 3(b), Table 3(b) shows the similar tendency as Table 3(a), but when the energy and frequency change, as the beam diameter is bigger, the ratio of sense is decreased in most of the sense area (for example: looking at $4 \mathrm{kHz}$ and $4.5 \mathrm{kHz}$ data when the energy is $150 \mu \mathrm{J}$, there was shown $45 \%$ and $40 \%$ of response rate in the beam diameter $0.46 \mathrm{~mm}$, but there was shown $25 \%$ and $30 \%$ of response rate in beam diameter $1.86 \mathrm{~mm}$ ). In addition, comparing Tables 3(a) and $3(b)$, in the same energy and frequency condition, as the beam diameter increases, the areas of dominant tactile changed into the areas of dominant sense of heat area (for example: looking at the 4 $\mathrm{kHz}$ and $4.5 \mathrm{kHz}$ data when the energy is $245 \mu \mathrm{J}$, the areas of dominant tactile in the beam diameter 
$0.46 \mathrm{~mm}$ changed into the areas of dominant sense of heat in the beam diameter $1.86 \mathrm{~mm}$ ).

In addition, when the beam diameter was $6.7 \mathrm{~mm}$, there was no area that more than $50 \%$ of subjects felt tactile in the same energy and frequency condition of the beam diameter $0.46 \mathrm{~mm}$ and $1.86 \mathrm{~mm}$. In general, the more energy increases, the ratio of sense increased in the same frequency condition. This phenomenon is considered because of that the bigger beam diameter causes the smaller energy density of laser beam. In addition, it could be seen that when the energy and frequency increased, the pain response rate increased as well.

Figure 3 is for examining the subject's response ratio due to frequency and energy change. As the diagrammatic result, Figure 3(a) shows the subjects' response in the frequency area condition of $3 \mathrm{kHz}$ $\sim 4.5 \mathrm{kHz}$ when the beam diameter was $0.46 \mathrm{~mm}$, Figure 3(b) shows the subjects' response in the frequency area condition of $3 \mathrm{kHz} \sim 4.5 \mathrm{kHz}$ when the beam diameter was $1.86 \mathrm{~mm}$, and Figure 3(c) shows the subjects' response in the frequency area condition of $3 \mathrm{kHz} \sim 4.5 \mathrm{kHz}$ when the beam diameter was $6.7 \mathrm{~mm}$. Looking at the result, it can be seen that when the beam diameter increases, the tactile response decreases. This phenomenon is considered that the decrease of energy density can cause the decrease of the temperature rise and the possibility of the receptor's action potential for feeling sense of heat, tactile, or pain. In other words, it can present the different feelings by the beam diameter change in the same energy and frequency condition through the laser.

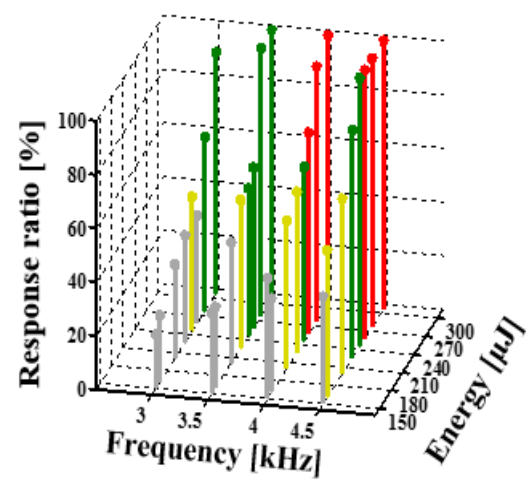

(a) Beam diameter $(0.46 \mathrm{~mm})$

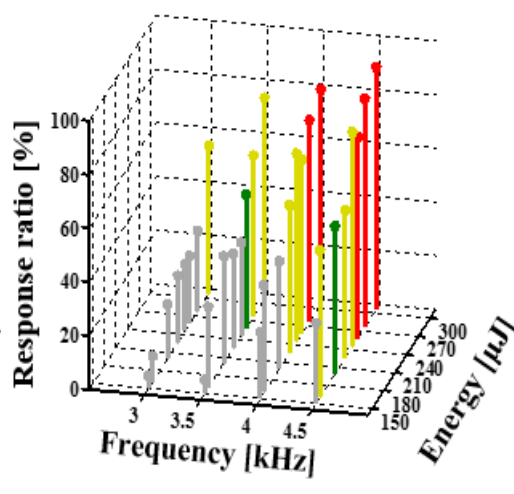

(b) Beam diameter $(1.86 \mathrm{~mm})$

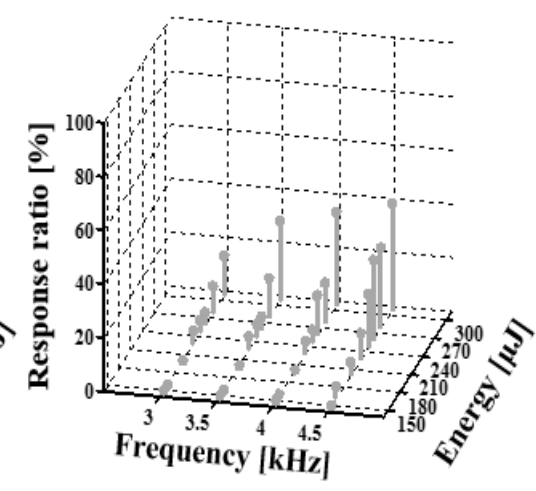

(c) Beam diameter $(6.7 \mathrm{~mm})$

Fig. 3. Change in the subjects' response rate by frequency and energy change.

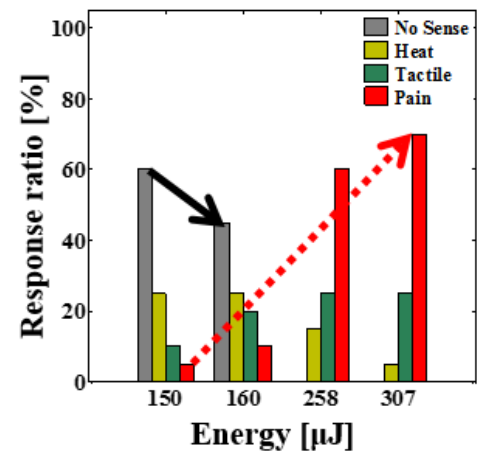

(a) Beam diameter $(0.46 \mathrm{~mm})$

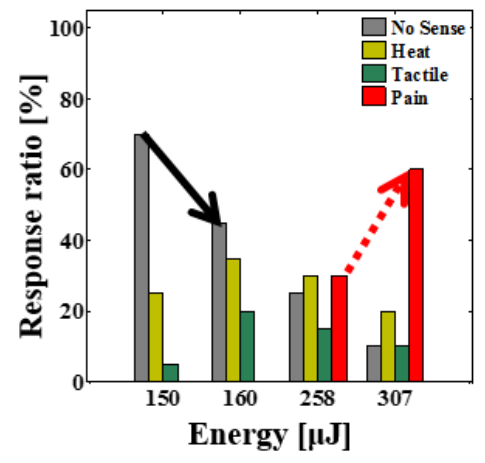

(b) Beam diameter $(1.86 \mathrm{~mm})$

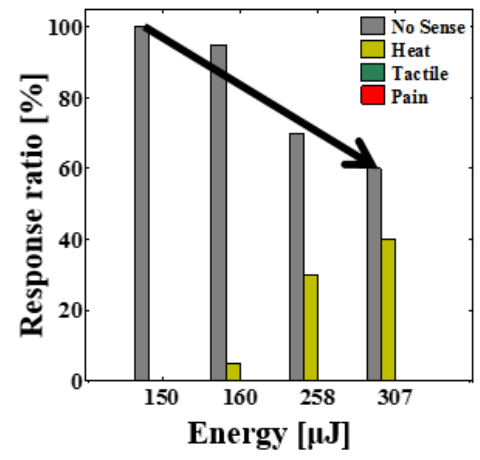

(c) Beam diameter $(6.7 \mathrm{~mm})$

Fig. 4. Responses by each sense according to changes in energy when the frequency was fixed (at frequency $=4.5 \mathrm{kHz}$ ). 
Figure 4 shows the sense response by the change of energy when the frequency was fixed in 4.5 $\mathrm{kHz}$. Figure 4(a) shows the result of the subjects' response by the energy change when the beam diameter was $0.46 \mathrm{~mm}$, Figure 4(b) shows the result of the subjects' response by the energy change when the beam diameter was $1.86 \mathrm{~mm}$, Figure 4(c) shows the result of the subjects' response by the energy change when the beam diameter was $6.7 \mathrm{~mm}$. In the result of experiment, the subjects who answered that felt the tactile and pain more when the energy increased. It could be seen when the energy increased, the ratio of tactile and pain increased as well in the beam diameter was $0.46 \mathrm{~mm}$. But in the same energy, the beam diameter increased as $1.86 \mathrm{~mm}$ and $6.7 \mathrm{~mm}$, so it could be seen that if the energy density decrease, the ratio of sense of heat will increase than tactile, and the response rate of pain will decrease. In other words, when the energy density decreases, the sense of heat than the tactile, the tactile than pain will increase in ratio. It is considered as the result of change in the temperature rise due to the change of absorption energy inside tissue by energy density difference.

\section{Conclusion}

This study found the approximate threshold of the senses that could be presented with laser by changing its various parameters and identified tactile responses. As a result of an experiment, frequency and energy increased, the ratio of feeling senses increased, and the dominant sense moved from the sense of heat through tactile sense to pain. Most senses generated by laser were accompanied by the sense of heat, and it felt from warm, got hot, and subject perceived it stinging or painful. At the same energy and frequency, the ratio of feeling senses decreased as the beam diameter increased.

It is judged that this phenomenon appeared as the ratio of temperature rise became low since the less energy density of the laser beam became. It was also found that there was a tendency with bigger beam diameter in which the areas with dominant tactile sense almost disappeared while many areas with the dominant sense of heat appeared. In the future, in order to find the sense of heat sensitivity by each part of the human body, an in-depth study would be necessary, and this paper will be the baseline data for studies of the senses of heat, tactile and pain, by adjusting frequency and energy, contribute to studying neurophysiology sector, and be applied to the basic clinical research etc.

\section{Acknowledgments}

This research was supported by the Pioneer Research Center Program through the National Research Foundation of Korea funded by the Ministry of Science, ICT \& Future planning (No.2011 0027920).

\section{References}

[1] E. Marchandise, A. Mouraux, L. Plaghki and F. Henrotte, Finite element analysis of thermal laser skin stimulation for a finer characterization of the nociceptive system, Journal of Neuroscience Methods 223 (2014), 1-10.

[2] F. Xu, T. Wen, K. Seffen and T. Lu, Modeling of skin thermal pain: A preliminary study, Applied Mathematics and Computation 205 (2008), 37-46.

[3] Q. Gu, L. Wang, F. Huang and W. Schwarz, Stimulation of TRPV1 by green laser light, Evidence-Based Complementary and Alternative Medicine 2012 (2012), 1-8.

[4] H.C. Kuo and L.J. Wu, Study on the prediction of temperature of ship plates and heat flux due to laser line heating using inverse and grey methods analysis, International Shipbuilding Progress 48 (2001), 227-245. 
[5] E.S. Albert, J.M. Bec, G. Desmadryl, K. Chekroud, C. Travo, S. Gaboyard, F. Bardin, I. Marc, M. Dumas, G. Lenaers, C. Hamel, A. Muller and C. Chabbert, TRPV4 channels mediate the infrared laser-evoked response in sensory neurons, Journal of Neurophysiology 107 (2012), 3227-3234.

[6] P. Parvin, H.R. Dehghanpour, M.S. Moghadam and V. Daneshafrooz, Validity of reciprocity rule on mouse skin thermal damage due to $\mathrm{CO}_{2}$ laser irradiation, Optics and Spectroscopy 115 (2013), 147-154.

[7] H. Athiraman, R.F. Wolf, K.E. Bartels, S. Shivakoti, H. Liu and W.R. Chen, Selective photothermal tissue interaction using 805-nm laser and indocyanine green in tissue welding, Journal of X-Ray Science and Technology 12 (2004), 117126.

[8] C.L. Ebbesen and H. Bruus, Analysis of laser-induced heating in optical neuronal guidance, Journal of Neuroscience Methods 209(1) (2012), pp. 168-177.

[9] A. Suppa, A. Biasiotta, D. Belvisi, L. Marsili, S. La Cesa, A. Truini, G. Cruccu and A. Berardelli, Heat-evoked experimental pain induces long-term potentiation-like plasticity in human primary motor cortex, Cerebral Cortex 23 (2013), 1942-1951.

[10] C.S. Madsen, B. Johnsen, A. Fuglsang-Frederiksen, T.S. Jensen and N.B. Finnerup, The effect of nerve compression and capsaicin on contact heat-evoked potentials related to A $\delta$-and C-fibers, Neuroscience 223 (2012), 92-101.

[11] K. Burnham, K. Schuster, A. Shingledecker, R. Kornegay and J. Oliver, Effect of laser thermal injury on langerhans cells in mouse and hairless guinea pig epidermis, Photochemistry and Photobiology 89 (2013), 1249-1254.

[12] G. Panick, H. Herberhold, Z. Sun and R. Winter, Heat, cold and pressure induced denaturation of proteins, Journal of Spectroscopy 17 (2003), 367-376.

[13] A.L. Pribisko and E.R. Perl, Use of a near-infrared diode laser to activate mouse cutaneous nociceptors in vitro, Journal of Neuroscience Methods 194 (2011), 235-241.

[14] F. Yang, Y. Cui, K. Wang and J. Zheng, Thermosensitive TRP channel pore turret is part of the temperature activation Pathway, Proceedings of the National Academy of Sciences 107 (2010), 7083-7088.

[15] A. Mouraux, M. Rage, D. Bragard and L. Plaghki, Estimation of intraepidermal fiber density by the detection rate of nociceptive laser stimuli in normal and pathological conditions, Neurophysiologie Clinique/Clinical Neurophysiology 42 (2012), 281-291. 